\title{
Twist regulates Yorkie to guide lineage reprogramming of syncytial alary muscles
}

\author{
Short running title: Reprogramming requires Twist \\ Marcel Rose ${ }^{1}$, Jakob Bartle-Schultheis ${ }^{1}$, Katrin Domsch ${ }^{1,2}$, Ingolf Reim¹, \\ Christoph Schaub ${ }^{*}$
}

${ }^{1}$ Friedrich-Alexander-Universität Erlangen-Nürnberg, Department of Biology, Division of Developmental Biology, Staudtstr. 5, 91058 Erlangen, Germany

${ }^{2}$ Heidelberg University, Centre for Organismal Studies (COS), Department of Developmental Biology, Im Neuenheimer Feld 230, 69120 Heidelberg, Germany

\section{*Author for correspondence: \\ https://orcid.org/0000-0003-3714-0050 \\ christoph.schaub@fau.de}

Key words: Twist, Yorkie, FGFR, muscle, lineage plasticity, reprogramming 


\section{Summary}

The genesis of syncytial muscles is typically considered as a paradigm for an irreversible developmental process. Notably, transdifferentiation of syncytial muscles is naturally occurring during Drosophila development. The ventral longitudinal heart-associated musculature (VLM) arises by a unique mechanism that revokes the differentiated fate from the so-called alary muscles and comprises at least two distinct steps: syncytial muscle cell fragmentation into single myoblasts and direct reprogramming into founder cells of the VLM lineage. Here we provide evidence that the mesodermal master regulator twist plays a key role during this reprogramming process. Acting downstream of Drosophila Tbx1 (Org-1) in the alary muscle lineage, Twist is crucially required for the derepression of the Hippo pathway effector Yki and thus for the initiation of syncytial muscle dedifferentiation and fragmentation. Subsequently, cell-autonomous FGFR-RasMAPK signaling in the resulting mono-nucleated myoblasts is maintaining Twist expression, thereby stabilizing nuclear Yki activity and inducing their lineage switch into the founder cells of the VLM.

\section{Introduction}

Lineage restriction is an essential concept in multicellular organisms. On the one hand, a lower fate commitment enables a high regenerative potential, but on the other hand, a higher fate commitment prevents uncontrolled growth and cancer. During Drosophila embryonic development early fate decisions within the blastoderm are initiated by the bHLH transcription factor Twist, which is crucial for mesoderm specification (Thisse et al., 1988) and is required for the activation of mesoderm specific factors (Furlong et al., 2001; Leptin, 1991) such as the Drosophila FGFR heartless ( $h t l$ ), required for mesodermal cell migration (Beiman et al., 1996; Gisselbrecht et al., 1996; Shishido et al., 1993; Shishido et al., 1997), and Mef2 initiating muscle differentiation (Bour et al., 1995; Lilly et al., 1995; Taylor et al., 1995). Although Twist was reported to promote embryonic myogenesis in Drosophila it appears to play the opposite role during vertebrate myogenesis, where primary Twist function is proposed in preventing the premature differentiation of myogenic cells (Baylies and Bate, 1996; Hebrok et al., 1994; Rohwedel et al., 1995; Spicer et al., 1996). 
Drosophila twist is known to become restricted after its early mesodermal expression to adult muscle precursors (AMPs), muscle stem cells from which the adult somatic musculature will arise during metamorphosis (Bate et al., 1991). Herein we report that twist expression additionally persists in the embryonic and larval heart-associated syncytial alary muscles (AMs), which connect the embryonic heart to internal organs and the exoskeleton (Bataillé et al., 2020; Boukhatmi et al., 2014). Our recent work has shown that during metamorphosis a subset of these AMs exhibits an extraordinary level of cell fate plasticity. Escaping histolysis, the three anterior muscle pairs undergo an in vivo direct lineage reprogramming process that includes their dedifferentiation, fragmentation into mononucleated alary muscle derived cells (AMDCs) and redifferentiation into the ventral longitudinal musculature of the heart (VLM) (Schaub et al., 2015). We demonstrate that after the onset of metamorphosis twist expression persists during AM to VLM transdifferentiation in the AM lineage and that the reprogramming process crucially depends on Twist function. Mechanistically, the induction of trans-differentiation depends on the function of the Drosophila homolog of Tbx1 (optomotor-blind-related-gene-1, org-1), whereas the initiation of syncytial muscle dedifferentiation and fragmentation requires derepression of the transcriptional effector of the Hippo pathway, Yorkie (Yki) (Schaub et al., 2015; Schaub et al., 2019). We find that twist expression during lineage reprogramming is maintained by Org-1 and that Twist function is required for the derepression of Yki function in the anterior AMs. This identifies Twist as a crucial factor that links these two mechanisms. We show that Twist together with the Drosophila FGFR Heartless is essential for the reprogramming process of the AMDCs into the founder myoblasts of the VLMs and demonstrate that this lineage switch requires the stabilization of nuclear Yki localization in the AMDCs by active RasMAPK signaling. Altogether, our results show that a Twist/Yki/FGFR axis plays a central role in the regulatory network that mediates syncytial muscle cell fate plasticity and initiates a lineage switch during cellular reprogramming. Of note, vertebrate orthologs of Twist, Yki and FGFR are well known to be involved in the reprogramming processes that transform myogenic cells into rhabdomyosarcoma (Goldstein et al., 2007; Li et al., 2019; Tremblay et al., 2014). Our results connect the function of this set of regulators within a naturally occurring muscle lineage reprogramming event. Apart from adding mechanistic insights into this process they are potentially also relevant for neoplastic reprogramming. 


\section{Results}

Twist is expressed in the alary muscle lineage and is required for AM to VLM lineage reprogramming

Twist expression is found during early embryonic development in the whole mesoderm, where its expression is regulated by upstream enhancer elements that are bound by the morphogen Dorsal (Jiang et al., 1991; Thisse et al., 1991). During later developmental stages somatic twist expression is known to be restricted to the AMPs that are specified via the rhomboid-triggered EGF signaling pathway (Bate et al., 1991; Figeac et al., 2010; Thisse et al., 1988). Unexpectedly, during an enhancer dissection analysis at the twist locus, reporter constructs driven by the complete downstream intergenic region (twist.Ko6-GFP, Fig. 1A) were found to include expression in the embryonic alary muscles (Dominik Müller, Jakob Bartle-Schultheis and Manfred Frasch, unpublished data; Fig. 1B). Visualization of the Twist protein together with an alary muscle-specific reporter (tupAME-GFP) during embryonic stages clearly show a localization of Twist in the nuclei of the embryonic alary muscles (Fig. 1C,C'). Moreover, twist.Ko6-GFP can be detected in larval alary muscles as well as in the VLM and the adult alary muscles (Fig. 1D-F). Live imaging demonstrated that the twist.Ko6-GFP reporter is active during the whole AM to VLM transdifferentiation process in the alary muscle lineage (video S1). The analysis of the expression pattern of a GFP-tagged version of Twist under endogenous control (twi::GFP) revealed that it is expressed in the org-1-RFP positive AMs, after dedifferentiation and fragmentation in the AMDCs as well as in the primordia of the VLM (Fig. 2A-C), indicating a potential role of Twist during alary muscle reprogramming. To dissect a possible requirement of twist function during VLM generation we induced its AM specific knock-down by RNAi or by inducible CRISPR using org-1-GAL4 during metamorphosis. The analyses of the resulting phenotypes revealed a strong perturbation of VLM formation and alary muscle reprogramming (Fig. 2E,H). Accordingly, live imaging demonstrated that upon RNAi mediated twist knock-down alary muscle dedifferentiation and fragmentation is severely impaired (video S2). Past work has shown that Twist function during embryonic myogenesis in Drosophila as well as vertebrates depends on dimer choice and protein-protein interactions (Franco et al., 2011). Interestingly, the downregulation of the Drosophila E-protein Daughterless, which has been proposed to inhibit somatic myogenesis via heterodimerization with Twist (Castanon et al., 2001), by RNAi does not cause a significant phenotype during AM lineage reprogramming (Fig. $2 \mathrm{H}$ ). 
In contrast, the elevation of the transcript levels of twist and nautilus, the Drosophila MyoD homolog (Michelson et al., 1990), using org-1-GAL4 during lineage reprogramming abolishes VLM formation (Fig. 2F,G,H), indicating a dose-dependent, mechanistical role for bHLH factor interaction during transdifferentiation. These results demonstrate an important role for Twist function in mediating lineage plasticity and reversal of differentiated, syncytial muscle fate during AM lineage programming.

\section{Twist links Org-1 and Yki function during AM dedifferentiation}

The induction of AM to VLM transdifferentiation crucially requires a transcriptional program that is activated by the AM lineage-specific induction of the Drosophila Tbx1 homolog Org-1 and its direct target, the Islet1 homolog Tailup (Schaub et al., 2015). To test for any functional connections between Org-1, Tup and Twist we analyzed the expression pattern of twi::GFP in genetic backgrounds that produce AM-specific org-1 or tup knock-downs using org-1-GAL4. Strikingly, loss of Org-1 or of Tup leads to the nearly complete abolishment of twi::GFP expression in the org-1-RFP positive alary muscles (Fig. $3 \mathrm{~B}, \mathrm{C})$, indicating that twist represents a downstream target of the Org-1 regulatory cascade during AM transdifferentiation. To further dissect the regulatory function of Org1 on the twist cis-regulatory region (CRM) we reanalyzed the twist.Ko6 downstream intergenic region and were able to identify an enhancer region that drives GFP reporter expression in the VLM lineage (twiVLM-GFP, Fig. 3D,F), suggesting a role of this CRM in twist regulation during AM lineage reprogramming. Org-1 as well as Tup are capable to significantly bind twiVLM in vivo (Fig. 3D) (Kudron et al., 2018) and the mutation of its predicted Org-1 binding sites (twiVLM-orgI-IIImut-GFP, Fig. 3E) leads to nearly complete abolishment of reporter activity in the VLM lineage (Fig 3G), thus demonstrating direct regulation of twist via this CRM by Org-1 during AM to VLM transdifferentiation.

Yki represents the transcriptional effector of the Hippo network and its function as a transcriptional co-activator of Scalloped (Sd) is regulated by phosphorylation, thereby inhibiting its nuclear translocation (Goulev et al., 2008; Huang et al., 2005; Wu et al., 2008; Zhao et al., 2008). During AM to VLM transdifferentiation, Org-1 and Tup induce the derepression of the Yki/Sd complex in the syncytial AMs and thereby the activation of its target genes, thus leading to the dedifferentiation of the AMs into mononucleated cells and the induction of lineage reprogramming (Schaub et al., 2019). To dissect a functional connection between Twist and Yki during AM transdifferentiation we forced the coexpression of a phosphorylation resistant, constitutively active version of Yki together 
with RNAi against twist by using org-1-GAL4. Strikingly, we observed that constitutively active Yki caused a significant rescue of VLM formation in Twist knock-down backgrounds (UAS-dsRNA-twi, UAS-yki.S168A; $\mathrm{p} \leq 0.01$; Fig. 4A-C), strongly suggesting that Twist is required for Yki derepression during alary muscle reprogramming. Taken together, these results support a mechanism that requires Org-1-dependent Twist function for the derepression of Yki in the alary muscles and concomitant activation of Yki/Sd target gene programs, which trigger alary muscle dedifferentiation.

\section{Nuclear Twist and Yki are stabilized by FGFR signaling during muscle lineage reprogramming}

During the establishment of the adult abdominal somatic musculature the Twist positive adult muscle precursors show expression of the Drosophila FGFR Heartless (Htl), which mediates the induction of founder myoblast fate in some of them (Dutta et al., 2005). Stainings for $h t l$ transcript as well as live imaging of the activity of a GAL4 line (htl-GAL4) (Jenett et al., 2012) driven by an $h t l$ enhancer containing previously characterized early embryonic Twist-binding sites (Stathopoulos et al., 2004; Zinzen et al., 2009), revealed that $h t l$ is expressed during AM to VLM transdifferentiation in the alary muscles as well as in the AMDCs (Fig. 4D,E; Fig. S1). Since after induction of RNAi against $h t l$ (UAS-dsRNA$h t l)$ or the forced expression of a dominant negative version of Htl (UAS- $h t l . D N)$ no org1-RFP positive muscles remain in pharate adults (Fig. 4A,F; Schaub et al. 2015), we deduce that activated FGFR signaling induces molecular events that are crucially required for alary muscle lineage reprogramming. To further investigate the role of FGFR signaling during AM to VLM transdifferentiation we performed live imaging experiments, thus demonstrating that mis-expression of a dominant negative version of Htl (UAS-htl.DN) using org-1-GAL4 has no influence on alary muscle dedifferentiation and fragmentation but inhibits reprogramming of the org-1-RFP positive AMDCs into the founder cells of the VLM (video S3). Since reprogramming of the AMDCs crucially depends on the activation of Yki targets (Schaub et al., 2019), we asked if Yki activity in the AMDCs may be connected with activated Htl signaling. Our analyses revealed that the loss of VLM phenotypes generated via the activation of $\mathrm{Htl}^{\mathrm{DN}}(U A S-h t l . D N)$ can be significantly rescued by co-expression of a constitutive active Yki (UAS-htl.DN; UAS-yki.S168A, $\mathrm{p} \leq 0.001$, Fig. $4 A, F, G)$, thus suggesting that activated FGFR signaling is required for the maintenance of nuclear Yki function during AMDC reprogramming. Interestingly, $\mathrm{Htl}{ }^{\mathrm{DN}}(U A S-h t l . D N)$ also leads to the loss of twi::GFP expression in the AMDCs, but not in the alary muscles before 
fragmentation (Fig. 4H,I). Taken into account that constitutive active Yki is epistatic over Twist (Fig. 4A,C), this suggests a regulatory interaction between FGFR, Twist and Yki function. Since it has been shown that Hippo signaling in wing discs can be negatively regulated via activated EGFR-Ras-MAPK signaling through the Ajuba LIM protein Jub (Reddy and Irvine, 2013), we speculated that sustained derepression of Yki in the AMDCs may depend on an analogous mechanism mediated by FGFR-Ras-MAPK signaling. Whereas induction of RNAi against EGFR or forced expression of the EGFR antagonist Argos has no phenotypic consequences (Fig. 4A), org-1-GAL4 induced expression of dominant negative versions of Drosophila Ras (UAS-Ras85D.DN) as well as mammalian Ras proteins (UAS-Ras.DN) or downregulation of the Drosophila MAPK Rolled (Rl, UASdsRNA-rl) lead to the loss of VLM formation and AMDC reprogramming (Fig 4A,J). Strikingly, these phenotypes can be significantly rescued by co-expression of phosphorylation resistant Yki during AMDC reprogramming $(\mathrm{p} \leq 0.001$, Fig. 4A,K), pointing towards a FGFR-Ras-MAPK-dependent regulation of nuclear Yki. The downregulation of Ajuba (UAS-dsRNA-jub) using org-1-GAL4 provokes the abolishment of VLM generation (Fig. 4A), but unexpectedly these phenotypes cannot be significantly rescued by the constitutive activation of Yki targets, suggesting additional roles of Jub during AMDC reprogramming. Thus, we propose a model in which FGFR-Ras-MAPK signaling is required for the stabilization of nuclear Twist and Yki during reprogramming of the AMDCs into the VLM founder cells.

\section{Discussion}

Twist function during embryonic muscle development in Drosophila has been described to possess dominant myogenic properties (Baylies and Bate, 1996), whereas vertebrate Twist is excluded from the myotome during embryogenesis and has been implicated in inhibiting myogenesis (Te and Reggiani, 2002). Twist expression and function in terminally differentiated striated, syncytial muscles has not been described yet. Here we show that Twist expression persist in the syncytial striated alary muscles after terminal differentiation and during metamorphosis in Drosophila. The alary muscles are a special type of striated muscles that are clearly distinguished from the body wall muscles (Bataillé et al., 2020; Boukhatmi et al., 2014), a feature that may be functionally connected to their persistent twist expression. During metamorphosis, the alary muscles demonstrate an extraordinary amount of cellular fate plasticity. Whereas the posterior 
larval alary muscles are remodeled into the adult alary muscles, the anterior ones generate the heart-associated ventral longitudinal musculature (VLM) by transdifferentiation (Schaub et al., 2015). Twist function and cellular plasticity have been connected in the literature mainly due to its role as one of the transcriptional master regulators during the transition from epithelial to mesenchymal states (EMT), crucially required for embryonic morphogenesis and often linked to metastasis in various cancer models (Lu and Kang, 2019). In terms of myogenic cell fate plasticity it is known that prolonged Twist expression negatively regulates muscle cell differentiation during embryonic myogenesis as well as adult flight muscle development in Drosophila (Anant et al., 1998; Cripps and Olson, 1998; Domsch et al., 2020), whereas forced expression of vertebrate Twist proteins can even reverse myogenic differentiation in vitro by unwiring the myogenic regulatory network from its targets (Hjiantoniou et al., 2008; Li et al., 2019; Liu et al., 2017; Mastroyiannopoulos et al., 2013) and appear to play a role during the regeneration of craniofacial muscles (Zhao et al., 2020). Here we provide evidence that Twist mediates the reversal of syncytial cell fate uncovering an unknown function of Twist in Drosophila muscles during metamorphosis.

It has been shown that sustained activation of the Hippo effector YAP inhibits the terminal myogenic differentiation program and that its Drosophila counterpart, Yki, induces reversal of syncytial differentiation during AM lineage reprogramming (Schaub et al., 2019; Sun et al., 2017; Watt et al., 2010). Here we demonstrate that Twist function is required for the derepression of Yki during AM transdifferentiation, connecting the Twist regulatory network to the activation of Yki target genes required for myogenic fate plasticity. Numerous studies have provided evidence for roles of YAP and TAZ, the vertebrate homologs of Yki, in EMT transdifferentiation and proposed that the induction of EMT upon forced Twist expression in cultured epithelial cells is connected to the inactivation of the Hippo pathway (Pan, 2010; Wang et al., 2016). These two mechanisms could be linked via the activation of JNK signaling and its transcriptional effector AP-1, both shown to be required for Twist-induced EMT as well as for Yki target activation (Nam et al., 2015; Sahu et al., 2015; Schaub et al., 2019; Sun and Irvine, 2013). Thus we speculate that Twist-induced abrogation of Yki target gene repression may represent a common molecular mechanism mediating cell fate plasticity in mesodermal cells.

Twist function and FGFR signaling have been shown to be closely connected during development of mesodermal derivatives in various species (Beiman et al., 1996; Bloch- 
Zupan et al., 2001; Gisselbrecht et al., 1996; Harfe et al., 1998; Johnson et al., 2000; Pfeifer et al., 2014; Shishido et al., 1993; Shishido et al., 1997). During the genesis of the Drosophila adult musculature, FGFR signaling is required in Twist positive myoblasts for the specification of the abdominal founder cells that will seed the respective myofibers (Dutta et al., 2004; Dutta et al., 2005). We show that induction of FGFR signaling is required in another population of Twist positive myoblasts, i. e. those derived from the fragmentation of Twist positive syncytial alary muscles (AMDCs) during metamorphosis, to induce their lineage switch to the founder cells of the ventral heart-associated musculature (VLM). Furthermore, our data link active FGFR/MAPK signaling with Twistmediated sustained de-repression of Yki in these cells. This connects Twist function to the induction of a potential Twist/Yki/FGFR feed-forward pathway driving AMDC lineage reprogramming. This is reminiscent of a mechanism proposed to be required for survival in cancer cells (Hua et al., 2016; Rizvi et al., 2016).

Taken together we propose a model in which Twist acts as a key regulator of alary muscle lineage reprogramming by coupling spatially and temporally specific transcriptional inputs to the de-repression of Yki targets (Fig. 4L). At the onset of metamorphosis, Org1/Tbx1 and Tup/Isl1 expression are induced in the anterior alary muscles by the combinatorial inputs of the Hox protein Ultrabithorax and the ligand bound receptor of Ecdysone (Schaub et al., 2015). This leads to the stabilization of Twist expression only in the AMs fated to undergo transdifferentiation. Subsequently, the integration of the Org1, Tup and Twist induced transcriptional networks leads to the de-repression of Yki/Sd target genes (e. g. $M y c$ ) required for the induction of muscle dedifferentiation and alary muscle fragmentation (Schaub et al., 2019). Once the alary muscles have fragmented into single myoblasts, the subsequent activation of FGFR/MAPK signaling in the AMDCs stabilizes the expression of Twist-induced Yki/Sd target genes, possibly via a regulatory Twi/Yki/FGFR feed-forward loop, and mediates reprogramming of the AMDCs into the founder cells of the VLM. 


\section{Materials and methods}

\section{Drosophila stocks and culture conditions}

Standard Drosophila melanogaster genetics were performed at $25{ }^{\circ} \mathrm{C}$. The following stocks were used: [org-1-GAL4, org-1-RFP; twi::GFP], [org-1-RFP; twist.Ko6-GFP], tupAMEGFP (Boukhatmi et al., 2014), [org-1-GAL4, org-1-RFP], [org-1-GAL4, org-1-RFP; handGFP], [org-1-GAL4, org-1-RFP; UAS-Cas9.P2], [org-1-GAL4, org-1-RFP; UAS-yki.S168A] (Schaub et al., 2019), UAS-dsRNA-org-1 (62953), UAS-dsRNA-tup (51763), UAS-dsRNA-twi (51164), UAS-htl.DN-B40; UAS-htl.DN.-B61 (5366), UAS-dsRNA-htl-2 (35024), UASdsRNA-EGFR-1 (60012), UAS-dsRNA-EGFR-2 (25781), UAS-argos.M; UAS-argos.M (5363), UAS-Ras85D.N17 (4845), UAS-Ras.N17 (4846), UAS-dsRNA-rl (34855), UAS-dsRNA-jub (32923), GMR93H07-htl-GAL4 (40669), UAS-His::RFP (derived from 56555) and twi::GFP (79615) were obtained from the Bloomington Drosophila Stock Center. UAS-dsRNA-htl-1 (6692) UAS-dsRNA-da-1 (51297), UAS-dsRNA-da-2 (51300) and UAS-dsRNA-da-3 (105259) were obtained from the Vienna Drosophila Resource Center (VDRC).

\section{Generation of transgenic Drosophila stocks}

To create UAS-t::gRNA-twi ${ }^{4 x}$ we utilized the system described in (Port and Bullock, 2016). Using 1 ng pCFD6 (Addgene, Cat. No: 73915) as template, we amplified PCR fragments with the respective primers containing the gRNA sequences (Table S1) and utilizing Q5 Hot Start High-Fidelity 2X Master Mix (New England Biolabs, Cat. No: M0494L). After gelpurification of the PCR fragments with the QIAquick Gel Extraction Kit (Qiagen, Cat. No: 28704), these were assembled with BbsI-HF (New England Biolabs, Cat. No: 3539S) digested pCFD6 backbone to generate $U A S$-t::gRNA-twi $i^{4 x}$ using the NEBuilder $®$ HiFi DNA Assembly Cloning Kit (New England Biolabs, Cat. No: R5520S), following the instructions provided by the manufacturers.

To create twiVLM-GFP, the respective genomic region (2R:23052698..23054095) was amplified from $y w$ DNA with the primers KpnI-twiVLM-fw (GGGGT ACCCCCAGTAAGGCAAATTGCTCAG) and XhoI-twiVLM-rev (CCGCTCGAGCGGAACTTGC CTTGTCCTTCGTC) utilizing Q5 Hot Start High-Fidelity 2X Master Mix (New England Biolabs, Cat. No: M0494L). The resulting PCR product was gel purified with the QIAquick Gel Extraction Kit (Qiagen, Cat. No: 28704) and cloned into KpnI/XhoI of pH-Stinger-AttB (Jin et al., 2013) using T4 DNA ligase (New England Biolabs, Cat. No: M2020L). 
For the analogous creation of twiVLM-orgI-IIImut-GFP the Org-1 binding sites within the twiVLM sequence were predicted with Target Explorer (Sosinsky et al., 2003) using a positional weight matrix generated by SELEX with Org-1 (Jin et al., 2013) and mutated via site-directed mutagenesis.

Transgenes of the constructs were generated by utilizing the phiC31/attP/attB system (Bischof et al., 2007) and performed by a commercial embryo injection service (BestGene Inc, Chino Hills, CA 91709, USA) to insert UAS-t::gRNA-twi ${ }^{4 x}$ at the attP40 landing site or to insert twiVLM-GFP as well as twiVLM-orgI-IIImut-GFP into the attP2 landing site. Potential transformants were selected by mini-white expression.

\section{Fluorescent antibody staining}

For antibody stainings of embryos the standard fixation protocol (Wieschaus and Nüsslein-Volhard, 1986) was applied. For dissection of pupal stages with microsurgery scissors the pupal cases were first removed and prefixation with 3,7 \% formaldehyde for 30 minutes was performed. Pharate adults were dissected without prefixation. The dissected animals were fixed in 3,7 \% formaldehyde for 10 minutes and were washed three times for 30 minutes in PBT (PBS + $0.1 \%$ Tween@ 20 ).

Fixed embryos and pupae were blocked in 10 \% Bovine Serum Albumin (Serva, Cat. No: 11930.04) for 1 hour. Samples were incubated for overnight (embryos) or two days (pupae) at $4{ }^{\circ} \mathrm{C}$ with the respective primary antibodies: Rabbit polyclonal anti-GFP (Rockland, Cat. No: 600-401-215, 1:500), Goat polyclonal anti-GFP (GeneTex Cat. No: GTX26673, 1:1000), Rabbit polyclonal anti-RFP (Rockland, Cat. No: 600-401-379S, 1:500), Rat polyclonal anti-Tropomyosin (MAC141, abcam, Cat. No: ab50567, 1:200), Rat polyclonal anti-Org-1 ((Schaub et al., 2012), 1:100), Guinea pig polyclonal anti-Twist (a gift of Katrin Domsch given by Eileen Furlong, 1:200), Rabbit polyclonal anti-Twist (a gift of Manfred Frasch, 1:500). To visualize filamentous actin Phalloidin conjugated either with Atto 488 (1:1000, Sigma-Aldrich, Cat. No: 49409) or Atto 647N (1:1000, SigmaAldrich, Cat. No: 65906) was added to the antibody solution. After the incubation with the primary antibodies the embryos and pupal dissections were washed three times for 30 minutes in PBT and incubated with the respective secondary antibodies: Alexa Fluor 488 conjugated Donkey Anti-Goat (abcam Cat. No: ab150129), Alexa Fluor 488 conjugated Goat Anti-Rabbit (ThermoFisher Scientific Cat. No: A-11008), DyLight 549 conjugated Goat anti-Rabbit (Jackson ImmunoResearch, Cat. No: 111-505-003) Alexa 
Fluor 555 conjugated Donkey Anti-Rabbit (abcam Cat. No: ab150074), Cy3 conjugated Donkey anti-Guinea pig (Jackson ImmunoResearch, Cat. No: 706-165-148), DyLight 649 conjugated Goat anti-Rat (Jackson ImmunoResearch, Cat. No: 112-495-003) in 1:200 dilution overnight at $4{ }^{\circ} \mathrm{C}$. Finally, the stained animals were washed three times for 30 minutes in PBT and were embedded into Vectashield containing DAPI (Vectorlabs, Cat. No: H-1200).

\section{In situ hybridization chain reaction}

To detect heartless mRNA in pupae we performed in situ hybridization chain reaction (HCR) as described in (Choi et al., 2018). For this purpose, we purchased a kit containing a custom $h t l$ DNA probe set and a fluorescently labelled DNA HCR amplifier as well as hybridization, wash and amplification buffers from Molecular Instruments (Los Angeles, CA 90041, USA).

For dissection of pupal stages with microsurgery scissors the pupal cases were first removed and prefixation with 3,7 \% formaldehyde for 30 minutes was performed. The dissected animals were fixed in 3,7 \% formaldehyde for 10 minutes, washed three times for 10 minutes in PBT (PBS + $0.1 \%$ Tween (C)20), followed by a 5 minute wash in $50 \%$ Methanol/PBT, a 5 minute wash in $100 \%$ Methanol and another 5 minute wash in $50 \%$ Methanol/PBT. The dissected pupae were rehydrated by three 5 minute washes in PBT followed by three 30 minute washes in PBT. After post-fixation with 3,7 \% formaldehyde for 30 minutes the specimens were washed for three times with PBT for 5 minutes. After 30 minutes of prehybridization at $37^{\circ} \mathrm{C}$ in hybridization buffer, dissected pupae were hybridized with 0,4 pmol of the $h t l$ DNA probe set in $100 \mu$ l hybridization buffer for 16 hours at $37^{\circ} \mathrm{C}$. To remove unbound probes, the specimens were washed four times for 15 minutes with probe wash buffer at $37^{\circ} \mathrm{C}$, followed by three washes with $5 \mathrm{xSSCT}$ $(5 \mathrm{xSSC}+0.1 \%$ Tween(C) 20$)$ at room temperature. For signal amplification the embryos and pupae were subjected to preamplification in amplification buffer for 30 minutes, followed by incubation with 6 pmol of each snap cooled, Alexa Fluor 647 labelled amplification hairpin in $100 \mu \mathrm{l}$ amplification buffer for 16 hours at room temperature. To remove excess hairpins, two washes with 5xSSCT for 5 minutes and two washes with 5xSSCT for 30 minutes were performed. The pupae stained for $h t l$ mRNA localization were subjected to antibody stainings as described above. 


\section{Sample analysis}

Confocal Z-stacks of fixed or in vivo specimen were acquired with a Leica SP5 II (10x/0.4 HC PL APO Air, 20x/0.7 HC PL APO Glycerol, 63x/1.3 HC PL APO Glycerol). Maximum Projections of the Z-stacks were performed with FIJI/ImageJ (v1.52v) (Schindelin et al., 2012).

\section{In vivo time-lapse imaging}

Stage P3 pupae of the respective genotypes were aligned on a strip of double-faced adhesive tape connected to a slide, covered with a drop of Halocarbon oil 700 (Halocarbon Products Corp, Sigma Aldrich Cat. No: H8898) and a coverslip. Time-lapse series were acquired on a Leica SP5 II confocal system using a HC PL APO 10x/0.4 Air. Acquisition was done over a time course of 48 hours with the following settings: Pinhole 1.4 AU, 1.2x optical zoom, scan speed $200 \mathrm{~Hz}$, Z-stack about 30-35 sections with a step size of 2-4 $\mu \mathrm{m}$ and time intervals of 10 min per stack. Movies were generated using Leica Application Suite X (LAS X, Leica Microsystems) and with FIJI/ImageJ (v1.52v) (Schindelin et al., 2012).

\section{Statistical analysis}

VLM phenotype frequency was quantified with living pharate stages of the respective genotypes that were aligned on a microscope slide. With the aid of a fluorescence equipped microscope (Nikon, Eclipse 80i) the org-1-RFP lineage marker in the living animals was visualized and phenotypes with fully differentiated VLM (proper aligned and elongated muscle fibers) were scored versus phenotypes ranging from missing VLM to defective VLM differentiation (no aligned muscle fibers). The Chi square test function of Microsoft Excel (Microsoft) was used for statistical analysis of the samples and statistical significance was defined by $\mathrm{p} \leq 0.05$. Fluorescent signal quantification and illustration was performed with FIJI/ImageJ (v1.52v) (Schindelin et al., 2012).

\section{Acknowledgements}

We thank the Drosophila community for providing us with antibodies and fly stocks and in particular Manfred Frasch (Friedrich-Alexander-Universität Erlangen-Nürnberg) for his gifts of unpublished materials. We are also grateful to the Bloomington Drosophila 
Stock Center and the Vienna Drosophila Resource Center for providing fly stocks as well as to the Optical Imaging Centre Erlangen (OICE) for their support and facilities.

\section{Competing interests}

The authors declare no competing financial interests.

\section{Author contributions}

Conceptualization: Christoph Schaub; Investigation: Marcel Rose, Jakob BartleSchultheis, Katrin Domsch, Ingolf Reim and Christoph Schaub ; Writing - Original Draft: Christoph Schaub; Writing - Review \& Editing: Marcel Rose, Jakob Bartle-Schultheis, Katrin Domsch, Ingolf Reim and Christoph Schaub; Funding Acquisition: Christoph Schaub; Supervision: Christoph Schaub

\section{Funding}

This work was funded by the Deutsche Forschungsgemeinschaft (DFG) (SCHA 2091/1-1 to C.S.).

\section{References}

Anant, S., Roy, S., and VijayRaghavan, K. (1998). Twist and Notch negatively regulate adult muscle differentiation in Drosophila. Development 125, 1361-1369.

Bataillé, L., Colombié, N., Pelletier, A., Paululat, A., Lebreton, G., Carrier, Y., Frendo, J.L., and Vincent, A. (2020). Alary muscles and thoracic alary-related muscles are atypical striated muscles involved in maintaining the position of internal organs. Development 147.

Bate, M., Rushton, E., and Currie, D.A. (1991). Cells with persistent twist expression are the embryonic precursors of adult muscles in Drosophila. Development 113, 79-89.

Baylies, M.K., and Bate, M. (1996). twist: a myogenic switch in Drosophila. Science 272, 1481-1484.

Beiman, M., Shilo, B.Z., and Volk, T. (1996). Heartless, a Drosophila FGF receptor homolog, is essential for cell migration and establishment of several mesodermal lineages. Genes Dev 10, 2993-3002.

Bischof, J., Maeda, R.K., Hediger, M., Karch, F., and Basler, K. (2007). An optimized transgenesis system for Drosophila using germ-line-specific phiC31 integrases. Proc Natl Acad Sci U S A 104, 3312-3317.

Bloch-Zupan, A., Hunter, N., Manthey, A., and Gibbins, J. (2001). R-twist gene expression during rat palatogenesis. Int J Dev Biol 45, 397-404.

Boukhatmi, H., Schaub, C., Bataille, L., Reim, I., Frendo, J.L., Frasch, M., and Vincent, A. (2014). An Org-1-Tup transcriptional cascade reveals different types of alary muscles connecting internal organs in Drosophila. Development 141, 3761-3771.

Bour, B.A., O'Brien, M.A., Lockwood, W.L., Goldstein, E.S., Bodmer, R., Taghert, P.H., Abmayr, S.M., and Nguyen, H.T. (1995). Drosophila MEF2, a transcription factor that is essential for myogenesis. Genes Dev 9, 730-741. 
Castanon, I., Von Stetina, S., Kass, J., and Baylies, M.K. (2001). Dimerization partners determine the activity of the Twist bHLH protein during Drosophila mesoderm development. Development 128, 3145-3159.

Choi, H.M.T., Schwarzkopf, M., Fornace, M.E., Acharya, A., Artavanis, G., Stegmaier, J., Cunha, A., and Pierce, N.A. (2018). Third-generation in situ hybridization chain reaction: multiplexed, quantitative, sensitive, versatile, robust. Development 145.

Cripps, R.M., and Olson, E.N. (1998). Twist is required for muscle template splitting during adult Drosophila myogenesis. Dev Biol 203, 106-115.

Domsch, K., Schroeder, J., Janeschik, M., Schaub, C., and Lohmann, I. (2020). The Hox transcription factor Ubx ensures somatic myogenesis by suppressing the mesodermal master regulator Twist. bioRxiv doi:10.1101/2020.02.24.963231.

Dutta, D., Anant, S., Ruiz-Gomez, M., Bate, M., and VijayRaghavan, K. (2004). Founder myoblasts and fibre number during adult myogenesis in Drosophila. Development 131, 3761-3772.

Dutta, D., Shaw, S., Maqbool, T., Pandya, H., and Vijayraghavan, K. (2005). Drosophila Heartless acts with Heartbroken/Dof in muscle founder differentiation. PLoS Biol 3, e337. Figeac, N., Jagla, T., Aradhya, R., Da Ponte, J.P., and Jagla, K. (2010). Drosophila adult muscle precursors form a network of interconnected cells and are specified by the rhomboid-triggered EGF pathway. Development 137, 1965-1973.

Franco, H.L., Casasnovas, J., Rodríguez-Medina, J.R., and Cadilla, C.L. (2011). Redundant or separate entities?--roles of Twist1 and Twist2 as molecular switches during gene transcription. Nucleic Acids Res 39, 1177-1186.

Furlong, E.E., Andersen, E.C., Null, B., White, K.P., and Scott, M.P. (2001). Patterns of gene expression during Drosophila mesoderm development. Science 293, 1629-1633.

Gisselbrecht, S., Skeath, J.B., Doe, C.Q., and Michelson, A.M. (1996). heartless encodes a fibroblast growth factor receptor (DFR1/DFGF-R2) involved in the directional migration of early mesodermal cells in the Drosophila embryo. Genes Dev 10, 3003-3017.

Goldstein, M., Meller, I., and Orr-Urtreger, A. (2007). FGFR1 over-expression in primary rhabdomyosarcoma tumors is associated with hypomethylation of a 5' CpG island and abnormal expression of the AKT1, NOG, and BMP4 genes. Genes Chromosomes Cancer 46, 1028-1038.

Goulev, Y., Fauny, J.D., Gonzalez-Marti, B., Flagiello, D., Silber, J., and Zider, A. (2008). SCALLOPED interacts with YORKIE, the nuclear effector of the hippo tumor-suppressor pathway in Drosophila. Curr Biol 18, 435-441.

Harfe, B.D., Vaz Gomes, A., Kenyon, C., Liu, J., Krause, M., and Fire, A. (1998). Analysis of a Caenorhabditis elegans Twist homolog identifies conserved and divergent aspects of mesodermal patterning. Genes Dev 12, 2623-2635.

Hebrok, M., Wertz, K., and Fuchtbauer, E.M. (1994). M-twist is an inhibitor of muscle differentiation. Dev Biol 165, 537-544.

Hjiantoniou, E., Anayasa, M., Nicolaou, P., Bantounas, I., Saito, M., Iseki, S., Uney, J.B., and Phylactou, L.A. (2008). Twist induces reversal of myotube formation. Differentiation 76, 182-192.

Hua, G., Lv, X., He, C., Remmenga, S.W., Rodabough, K.J., Dong, J., Yang, L., Lele, S.M., Yang, P., Zhou, J., et al. (2016). YAP induces high-grade serous carcinoma in fallopian tube secretory epithelial cells. Oncogene 35, 2247-2265.

Huang, J., Wu, S., Barrera, J., Matthews, K., and Pan, D. (2005). The Hippo signaling pathway coordinately regulates cell proliferation and apoptosis by inactivating Yorkie, the Drosophila Homolog of YAP. Cell 122, 421-434. 
Jenett, A., Rubin, G.M., Ngo, T.T., Shepherd, D., Murphy, C., Dionne, H., Pfeiffer, B.D., Cavallaro, A., Hall, D., Jeter, J., et al. (2012). A GAL4-driver line resource for Drosophila neurobiology. Cell Rep 2, 991-1001.

Jiang, J., Kosman, D., Ip, Y.T., and Levine, M. (1991). The dorsal morphogen gradient regulates the mesoderm determinant twist in early Drosophila embryos. Genes Dev 5, 1881-1891.

Jin, H., Stojnic, R., Adryan, B., Ozdemir, A., Stathopoulos, A., and Frasch, M. (2013). Genome-wide screens for in vivo Tinman binding sites identify cardiac enhancers with diverse functional architectures. PLoS Genet 9, e1003195.

Johnson, D., Iseki, S., Wilkie, A.O., and Morriss-Kay, G.M. (2000). Expression patterns of Twist and Fgfr1, -2 and -3 in the developing mouse coronal suture suggest a key role for twist in suture initiation and biogenesis. Mech Dev 91, 341-345.

Kudron, M.M., Victorsen, A., Gevirtzman, L., Hillier, L.W., Fisher, W.W., Vafeados, D., Kirkey, M., Hammonds, A.S., Gersch, J., Ammouri, H., et al. (2018). The ModERN Resource: Genome-Wide Binding Profiles for Hundreds of Drosophila and Caenorhabditis elegans Transcription Factors. Genetics 208, 937-949.

Leptin, M. (1991). twist and snail as positive and negative regulators during Drosophila mesoderm development. Genes Dev 5, 1568-1576.

Li, S., Chen, K., Zhang, Y., Barnes, S.D., Jaichander, P., Zheng, Y., Hassan, M., Malladi, V.S., Skapek, S.X., Xu, L., et al. (2019). Twist2 amplification in rhabdomyosarcoma represses myogenesis and promotes oncogenesis by redirecting MyoD DNA binding. Genes Dev 33, 626-640.

Lilly, B., Zhao, B., Ranganayakulu, G., Paterson, B.M., Schulz, R.A., and Olson, E.N. (1995). Requirement of MADS domain transcription factor D-MEF2 for muscle formation in Drosophila. Science 267, 688-693.

Liu, N., Garry, G.A., Li, S., Bezprozvannaya, S., Sanchez-Ortiz, E., Chen, B., Shelton, J.M., Jaichander, P., Bassel-Duby, R., and Olson, E.N. (2017). A Twist2-dependent progenitor cell contributes to adult skeletal muscle. Nat Cell Biol 19, 202-213.

Lu, W., and Kang, Y. (2019). Epithelial-Mesenchymal Plasticity in Cancer Progression and Metastasis. Dev Cell 49, 361-374.

Mastroyiannopoulos, N.P., Antoniou, A.A., Koutsoulidou, A., Uney, J.B., and Phylactou, L.A. (2013). Twist reverses muscle cell differentiation through transcriptional downregulation of myogenin. Biosci Rep 33.

Michelson, A.M., Abmayr, S.M., Bate, M., Arias, A.M., and Maniatis, T. (1990). Expression of a MyoD family member prefigures muscle pattern in Drosophila embryos. Genes Dev 4, 2086-2097.

Nam, E.H., Lee, Y., Moon, B., Lee, J.W., and Kim, S. (2015). Twist1 and AP-1 cooperatively upregulate integrin $\alpha 5$ expression to induce invasion and the epithelial-mesenchymal transition. Carcinogenesis 36, 327-337.

Pan, D. (2010). The hippo signaling pathway in development and cancer. Dev Cell 19, 491505.

Pfeifer, K., Schaub, C., Domsch, K., Dorresteijn, A., and Wolfstetter, G. (2014). Maternal inheritance of twist and analysis of MAPK activation in embryos of the polychaete annelid Platynereis dumerilii. PLoS One 9, e96702.

Port, F., and Bullock, S.L. (2016). Augmenting CRISPR applications in Drosophila with tRNA-flanked sgRNAs. Nat Methods 13, 852-854.

Reddy, B.V., and Irvine, K.D. (2013). Regulation of Hippo signaling by EGFR-MAPK signaling through Ajuba family proteins. Dev Cell 24, 459-471. 
Rizvi, S., Yamada, D., Hirsova, P., Bronk, S.F., Werneburg, N.W., Krishnan, A., Salim, W., Zhang, L., Trushina, E., Truty, M.J., et al. (2016). A Hippo and Fibroblast Growth Factor Receptor Autocrine Pathway in Cholangiocarcinoma. J Biol Chem 291, 8031-8047.

Rohwedel, J., Horak, V., Hebrok, M., Fuchtbauer, E.M., and Wobus, A.M. (1995). M-twist expression inhibits mouse embryonic stem cell-derived myogenic differentiation in vitro. Exp Cell Res 220, 92-100.

Sahu, S.K., Garding, A., Tiwari, N., Thakurela, S., Toedling, J., Gebhard, S., Ortega, F., Schmarowski, N., Berninger, B., Nitsch, R., et al. (2015). JNK-dependent gene regulatory circuitry governs mesenchymal fate. EMBO J 34, 2162-2181.

Schaub, C., Marz, J., Reim, I., and Frasch, M. (2015). Org-1-dependent lineage reprogramming generates the ventral longitudinal musculature of the Drosophila heart. Curr Biol 25, 488-494.

Schaub, C., Nagaso, H., Jin, H., and Frasch, M. (2012). Org-1, the Drosophila ortholog of Tbx1, is a direct activator of known identity genes during muscle specification. Development 139, 1001-1012.

Schaub, C., Rose, M., and Frasch, M. (2019). Yorkie and JNK revert syncytial muscles into myoblasts during Org-1-dependent lineage reprogramming. J Cell Biol 218, 3572-3582.

Schindelin, J., Arganda-Carreras, I., Frise, E., Kaynig, V., Longair, M., Pietzsch, T., Preibisch, S., Rueden, C., Saalfeld, S., Schmid, B., et al. (2012). Fiji: an open-source platform for biological-image analysis. Nat Methods 9, 676-682.

Shishido, E., Higashijima, S., Emori, Y., and Saigo, K. (1993). Two FGF-receptor homologues of Drosophila: one is expressed in mesodermal primordium in early embryos. Development 117, 751-761.

Shishido, E., Ono, N., Kojima, T., and Saigo, K. (1997). Requirements of DFR1/Heartless, a mesoderm-specific Drosophila FGF-receptor, for the formation of heart, visceral and somatic muscles, and ensheathing of longitudinal axon tracts in CNS. Development 124, 2119-2128.

Sosinsky, A., Bonin, C.P., Mann, R.S., and Honig, B. (2003). Target Explorer: An automated tool for the identification of new target genes for a specified set of transcription factors. Nucleic Acids Res 31, 3589-3592.

Spicer, D.B., Rhee, J., Cheung, W.L., and Lassar, A.B. (1996). Inhibition of myogenic bHLH and MEF2 transcription factors by the bHLH protein Twist. Science 272, 1476-1480.

Stathopoulos, A., Tam, B., Ronshaugen, M., Frasch, M., and Levine, M. (2004). pyramus and thisbe: FGF genes that pattern the mesoderm of Drosophila embryos. Genes Dev 18, 687699.

Sun, C., De Mello, V., Mohamed, A., Ortuste Quiroga, H.P., Garcia-Munoz, A., Al Bloshi, A., Tremblay, A.M., von Kriegsheim, A., Collie-Duguid, E., Vargesson, N., et al. (2017). Common and Distinctive Functions of the Hippo Effectors Taz and Yap in Skeletal Muscle Stem Cell Function. STEM CELLS 35, 1958-1972.

Sun, G., and Irvine, K.D. (2013). Ajuba family proteins link JNK to Hippo signaling. Sci Signal 6, ra81.

Taylor, M.V., Beatty, K.E., Hunter, H.K., and Baylies, M.K. (1995). Drosophila MEF2 is regulated by twist and is expressed in both the primordia and differentiated cells of the embryonic somatic, visceral and heart musculature. Mech Dev 50, 29-41.

Te, K.G., and Reggiani, C. (2002). Skeletal muscle fibre type specification during embryonic development. J Muscle Res Cell Motil 23, 65-69.

Thisse, B., Stoetzel, C., Gorostiza-Thisse, C., and Perrin-Schmitt, F. (1988). Sequence of the twist gene and nuclear localization of its protein in endomesodermal cells of early Drosophila embryos. EMBO J 7, 2175-2183. 
Thisse, C., Perrin-Schmitt, F., Stoetzel, C., and Thisse, B. (1991). Sequence-specific transactivation of the Drosophila twist gene by the dorsal gene product. Cell 65,1191 1201.

Tremblay, A.M., Missiaglia, E., Galli, G.G., Hettmer, S., Urcia, R., Carrara, M., Judson, R.N., Thway, K., Nadal, G., Selfe, J.L., et al. (2014). The Hippo transducer YAP1 transforms activated satellite cells and is a potent effector of embryonal rhabdomyosarcoma formation. Cancer Cell 26, 273-287.

Wang, Y., Liu, J., Ying, X., Lin, P.C., and Zhou, B.P. (2016). Twist-mediated Epithelialmesenchymal Transition Promotes Breast Tumor Cell Invasion via Inhibition of Hippo Pathway. Sci Rep 6, 24606.

Watt, K.I., Judson, R., Medlow, P., Reid, K., Kurth, T.B., Burniston, J.G., Ratkevicius, A., De Bari, C., and Wackerhage, H. (2010). Yap is a novel regulator of C2C12 myogenesis. Biochem Biophys Res Commun 393, 619-624.

Wieschaus, E., and Nüsslein-Volhard, C. (1986). Looking at embryos. In Drosophila: a practical approach, R. DB, ed. (Oxford: IRL Press), pp. 199-227.

Wu, S., Liu, Y., Zheng, Y., Dong, J., and Pan, D. (2008). The TEAD/TEF family protein Scalloped mediates transcriptional output of the Hippo growth-regulatory pathway. Dev Cell 14, 388-398.

Zhao, B., Ye, X., Yu, J., Li, L., Li, W., Li, S., Yu, J., Lin, J.D., Wang, C.Y., Chinnaiyan, A.M., et al. (2008). TEAD mediates YAP-dependent gene induction and growth control. Genes Dev $22,1962-1971$.

Zhao, Y., Louie, K.W., Tingle, C.F., Sha, C., Heisel, C.J., Unsworth, S.P., Kish, P.E., and Kahana, A. (2020). Twist3 is required for dedifferentiation during extraocular muscle regeneration in adult zebrafish. PLoS One 15, e0231963.

Zinzen, R.P., Girardot, C., Gagneur, J., Braun, M., and Furlong, E.E. (2009). Combinatorial binding predicts spatio-temporal cis-regulatory activity. Nature 462, 65-70.

\section{Figure legends}

Figure 1: The bHLH transcription factor Twist is expressed in the syncytial alary muscles

(A) Schematic depiction of the genomic region used to create twist.Ko6-GFP. (B-B') In vivo live imaging of an embryo carrying twist.Ko6-GFP and org-1-HN18-RFP (org-1-RFP). Strong GFP expression can be detected in the first three pairs of org-1-RFP positive alary muscles (asterisks). (C-C') Dorsal view of an embryo carrying the alary muscle specific tupAME-GFP reporter and stained for Twist, Org-1 and GFP proteins. (C) Twist and Org-1 are clearly co-expressed in nuclei of the ( $\mathbf{C}^{\prime}$ ) anterior three pairs of alary muscles (asterisks) as well as the posterior ones marked by the expression of tupAME-GFP. The magnified views show a single anterior alary muscle. (D-D') In vivo live imaging of a dorsal view of a L3 larva carrying twist.Ko6-GFP and org-1-HN18-RFP. (D) twist.Ko6 drives GFP expression in the thoracic alary related muscles (TARMs) and the first three pairs of (D') org-1-RFP positive alary muscles. (E-E') Visualization of the expression 
pattern of org-1-RFP and twist.Ko6-GFP in a dissected late L3 stage. RFP and GFP are colocalized in the anterior alary muscles (asterisks). (F,F') twist.Ko6-GFP and org-1-RFP drive reporter expression in the ventral longitudinal musculature (VLM) attached to the adult heart of a stage P13 pharate fly. Twist-Ko6-GFP expression is additionally detected in the adult alary muscles (diamonds). Scale bars in D: $500 \mu \mathrm{m}$, Scale bars in E-F: $100 \mu \mathrm{m}$. Actin is visualized with phalloidin. DNA visualized with DAPI.

\section{Figure 2: Twist is required for the lineage reprogramming of alary muscles into the VLM.}

(A-C) Visualization of the org-1-RFP lineage marker and an endogenously GFP tagged version of twist (twi::GFP). twi::GFP can be detected (A-A') at the onset of metamorphosis in the nuclei of the syncytial alary muscles (AM) and (B-B') during reprogramming in the nuclei of the formed mononuclear cells (AMDCs) as well as (C-C') in the cells of the differentiating VLM. (D) org-1-RFP drives reporter expression in the ventral longitudinal musculature. (E) Induction of CRISPR in the alary muscles with HN39-org-1-GAL4 (org-1GAL4) against twist (org-1>>Cas9; t::gRNA-twi ${ }^{4 x}$ ) blocks VLM formation and prevents AM fragmentation (arrowheads). (F-G) HN39-org-1-GAL4 (org-1-GAL4) induced elevation of transcript levels of (F) twist (org-1>>twi) and (G) nautilus (org-1>>nau) leads to the abolishment of VLM formation. (H) Frequencies of observed VLM differentiation in the different genetic backgrounds. org-1-GAL4-induced twist RNAi (dsRNA-twi, $\mathrm{n}=111$, $\mathrm{p} \leq 0.001$ ) or CRISPR (Cas9P2; t::gRNA-twi, $\mathrm{n}=64, \mathrm{p} \leq 0.001$ ) abolishes significantly alary muscle transdifferentiation and VLM generation. Scale bars in A-C: $25 \mu \mathrm{m}$. Scale bars in D-E: $100 \mu \mathrm{m}$. Actin is visualized with phalloidin. DNA visualized with DAPI.

\section{Figure 3: Twist expression during lineage reprogramming depends on Org-1 and Tup}

(A-A") twi::GFP can be detected in the nuclei of the org-1-RFP positive alary muscles (AM) (B,C) Induction of RNAi with HN39-org-1-GAL4 (org-1-GAL4) against (B-B') Org-1 (org1 >>dsRNA-org-1) or (C-C") Tailup (Tup) (org-1 >>dsRNA-tup) leads to abolishment of twi::GFP expression in the alary muscles (AM). The indicated regions were used to quantify the expression level of nuclear DAPI, the $\mathrm{org}$-1-RFP lineage marker and twi::GFP in (A", B',C') in the respective genetic backgrounds. (D) Illustration of the twist downstream genomic region showing Tup (red) and Org-1 (green) binding as well as called peaks during embryonic development (Kudron et al., 2018). The region used for 
the generation of twiVLM-GFP is indicated. (E) Schematic depiction of the twiVLM enhancer region and of the Org-1 binding motif. The predicted Org-1 binding sites and the point mutations that have been introduced to create twiVLM-orgI-IIImut are indicated. (F-F") twiVLM-GFP drives reporter expression in the VLM. (G-G'). The mutation of the predicted Org-1 binding sites in twiVLM (twiVLM-orgI-III-GFP) leads to the downregulation of GFP reporter expression in the VLM. The regions used for the quantification of GFP expression in (F',G') are indicated. Actin is visualized with phalloidin. DNA visualized with DAPI. Scale bars in A-C: $25 \mu \mathrm{m}$. Scale bars in F-G: $100 \mu \mathrm{m}$.

Figure 4: A Twist/Yki/FGFR regulatory axis mediates the induction of alary muscle fragmentation and the lineage switch during AMDC reprogramming.

(A) Frequencies of observed VLM differentiation in the different genetic backgrounds. Co-expression of $\mathrm{Yki}^{\mathrm{S} 168 \mathrm{~A}}$ can significantly rescue the phenotypes provoked by downregulation of Twist $(n=39, p \leq 0.01)$ as well as forced expression of $\operatorname{Htl}^{\mathrm{DN}}(\mathrm{n}=54$, $\mathrm{p} \leq 0.001)$, Ras85D $\mathrm{D}^{\mathrm{DN}}(\mathrm{n}=74, \mathrm{p} \leq 0.001)$, mammalian $\operatorname{Ras}^{\mathrm{DN}}(\mathrm{n}=66, \mathrm{p} \leq 0.001)$ and knockdown of MAPK/Rolled (Rl) (n=36, p $\leq 0.001)$. (B) Induction of RNAi against twist with org-1GAL4 (org-1>>dsRNA-twi) disrupts VLM differentiation and AM transdifferentiation. (C) VLM generation can be rescued by co-expression of phosphorylation resistant hippo pathway effector Ykis168A in twist loss-of-function background (org-1 >>dsRNA-twi; $y k i . S 168 A$ ). (D) heartless ( $h t l$ ) transcript can be detected in the org-1-RFP positive alary muscles (AM) at the onset of transdifferentiation. (E) Live imaging of flies carrying $h t l$ GAL4 and UAS-His::RFP in addition to twi.Ko6-GFP. Arrowheads indicate htl-GAL4-driven expression of Histone-RFP in GFP positive AMDCs. (F) Forced expression of a dominant negative version of Htl (org-1>>htl.DN) abolishes VLM differentiation. (G) These phenotypes can be significantly rescued by co-expression of $\mathrm{Yki}^{\mathrm{S} 168 \mathrm{~A}}$ in $\mathrm{Htl}^{\mathrm{DN}}$ background (org-1>>htl.DN; yki.S168A). (H-H") org-1-GAL4 mediated induction of a dominant negative version of Htl ( $\mathrm{org}-1>>$ htl.DN) has no effect on nuclear localization of GFP tagged Twist (twi::GFP) in alary muscles (AM) but (I-I") leads to abolishment of the twi::GFP expression in the AMDCs. (H",I') The indicated regions were used to quantify the expression level of nuclear DAPI, the org-1-RFP lineage marker as well as twi::GFP in the respective genetic backgrounds. (J) Induction of RNAi against MAPK/Rl with org-1-GAL4 (org-1 >>dsRNA-rl) disrupts VLM differentiation. (K) The phenotypes provoked by Rl lossof-function can be rescued by co-expression of phosphorylation resistant Yki ${ }^{\mathrm{S} 168 \mathrm{~A}}$ ( org1 >>dsRNA-rl; yki.S168A). (L) Schematic depiction of regulatory interactions during AM to 
VLM transdifferentiation. Continuous lines indicate direct regulation, dashed lines indicate indirect regulation and question marks proposed regulatory interaction. Twist expression is induced by the Ultrabithorax (Ubx)/Ecdysone Receptor (EcR)/Org-1/Tup transcriptional framework, thus leading to the de-repression of Yki/Sd and resulting in the activation of targets (e. g. Myc) required for fragmentation of the alary muscles. In the alary muscle derived cells (AMDCs), the cell autonomous activation of FGFR-MAPK signaling leads to the stabilization of Twist expression. This leads to the sustained derepression of $\mathrm{Yki} / \mathrm{Sd}$, inducing the reprogramming process and the lineage switch by the activation of the VLM founder cell program. Scale bars in B,C\&F,G\&J,K: $100 \mu \mathrm{m}$. Scale bars in D,E: $50 \mu \mathrm{m}$. Scale bars in H,I: $25 \mu \mathrm{m}$. Actin is visualized with phalloidin. DNA is visualized with DAPI.

\section{Supplemental information}

\section{Figure S1.}

Activity of $h t l$ expression during AM/VLM transdifferentiation. Flies carrying $h t l-$ GAL4 (GMR93H07) and UAS-His::RFP in addition to twi.Ko6-GFP were imaged during metamorphosis. A-J show stills from the same image series in developmental order (dorsal view). Arrows indicate $h t l-G A L 4$-driven expression of Histone-RFP in (A, B) either AMs prior to abdominal compaction or (C-J) in AMDCs and the developing VLM. (Note that only some portions are optically accessible from the outside particularly at early pre-pupal stages.) Expression is also observed in the central heart and various flanking abdominal skeletal muscles (both GFP-negative) as well as the developing flight muscles (GFP-positive, to the left in C-J).

\section{Video S1.}

Dorsal view of a pupa that carries twist.Ko6-GFP and org-1-RFP. The movie starts at late stage P3 and demonstrates the co-expression of twist.Ko6-GFP and org-1-RFP during the transdifferentiation process of the larval alary muscles into the ventral longitudinal musculature of the heart. At the beginning of the movie, the syncytial org-1-RFP and twist.Ko6-GFP positive anterior alary muscles initiate dedifferentiation into mesenchymal, mononucleated myoblasts. These keep close contact to each other and migrate towards the dorsal vessel where they start re-differentiating into the ventral longitudinal heart muscles elongating along the heart tube towards the posterior. In addition, 
twist.Ko6-GFP can be detected in the remodeling org-1-RFP negative larval posterior alary muscles, the dorsal adult muscle precursors (AMPs) as well as the hindgut musculature.

\section{Video S2.}

Dorsal view of a pupa that carries hand-GFP and org-1-RFP and in which org-1-GAL4 mediated RNAi against twist was induced (org-1>>UAS-dsRNA-twi). The movie starts at late stage P3 and demonstrates that knock-down of twi prevents the alary muscles from dedifferentiating into mononucleated myoblasts, suggesting that Twist function is indispensable for the induction of this essential process during alary muscle transdifferentiation.

\section{Video S3.}

Dorsal view of a pupa that carries hand-GFP and org-1-RFP and in which a dominant negative version of the Drosophila FGFR Htl was induced (org-1 >>UAS-htl.DN). The movie starts at late stage P3 and shows that interference with FGFR mediated signaling has no impact on the fragmentation of the alary muscles into mononucleated myoblasts but demonstrates that transdifferentiation is arrested under these conditions after alary muscle dedifferentiation. This suggests an essential role of Htl-signaling during the lineage reprogramming of the alary muscle derived myoblasts into the founders of the VLM during transdifferentiation. 


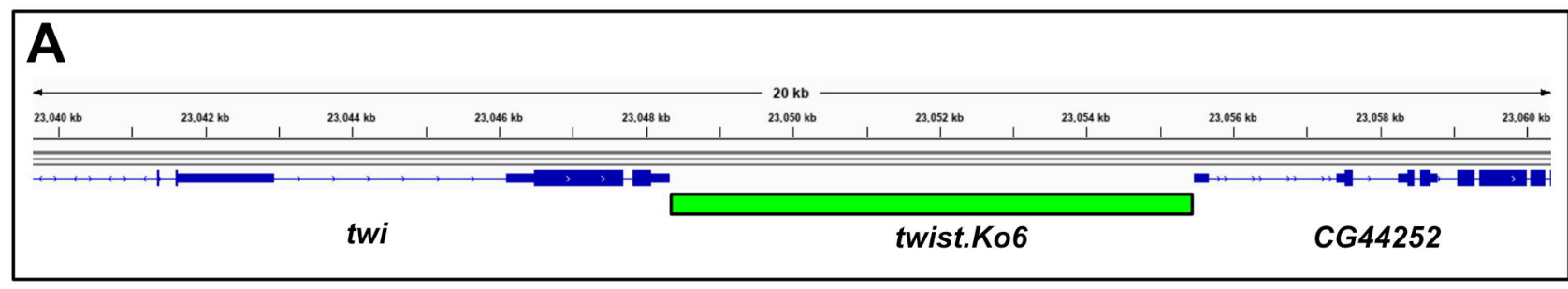

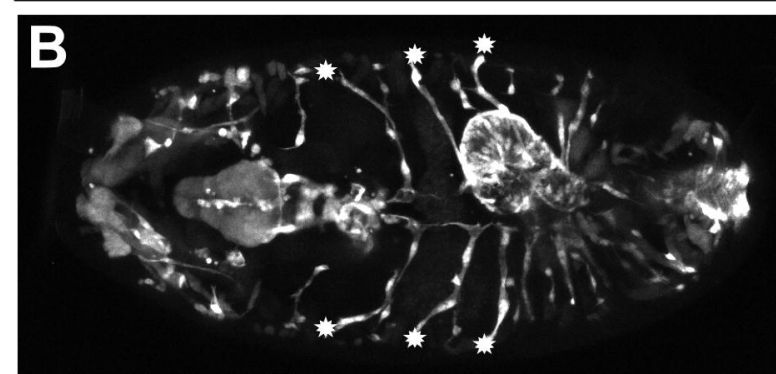

twist.Ko6-GFP
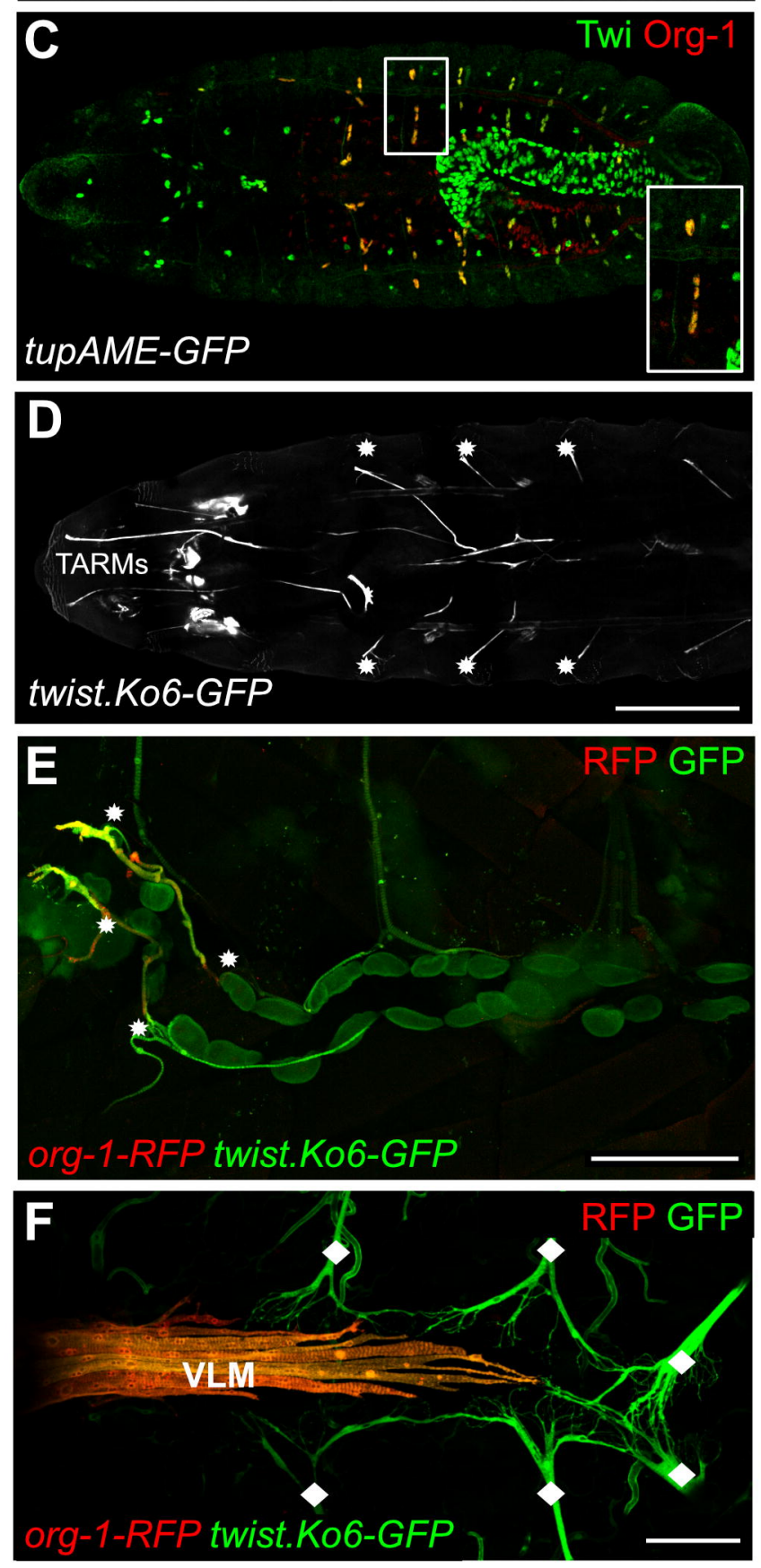

tupAME-GFP
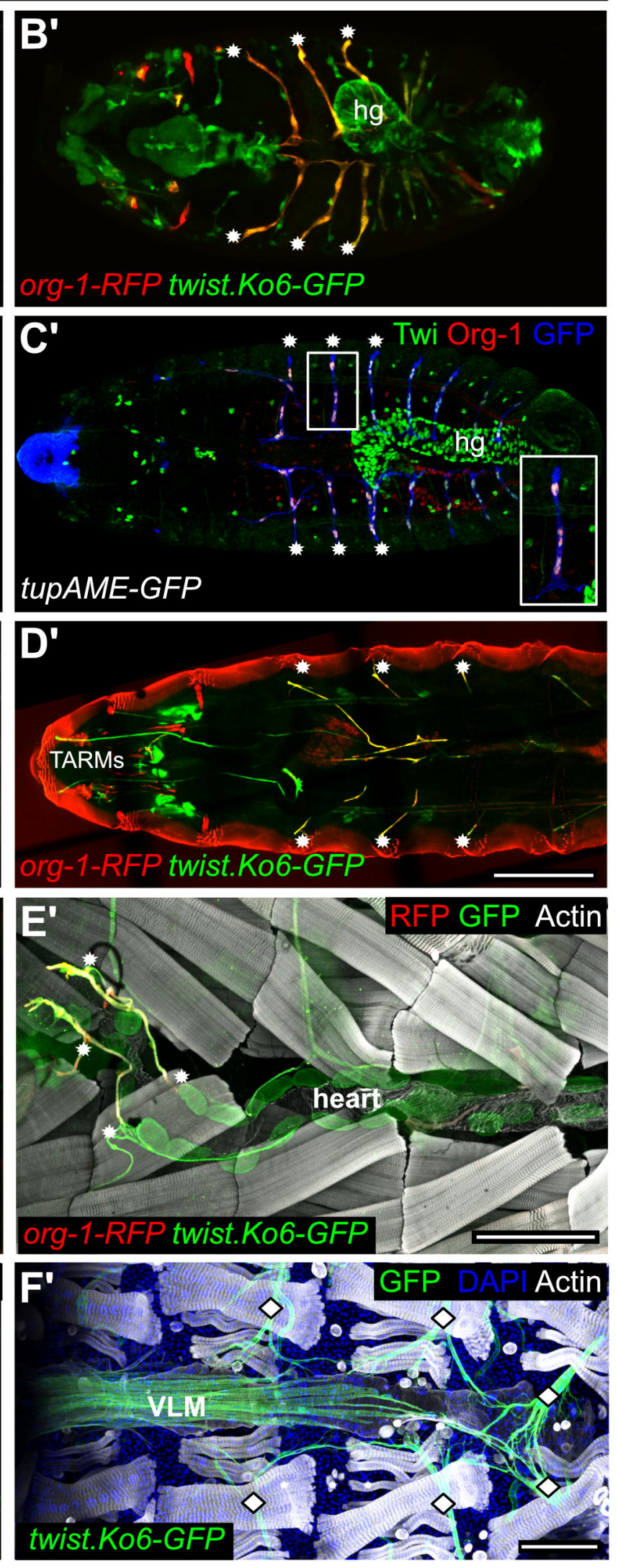
bioRxiv preprint doi: https://doi.org/10.1101/2020.06.22.165506; this version posted July 15, 2020. The copyright holder for this preprint which was not certified hv neer review/ is the author/funder. All riahts reserved No reuse allowed without permission.
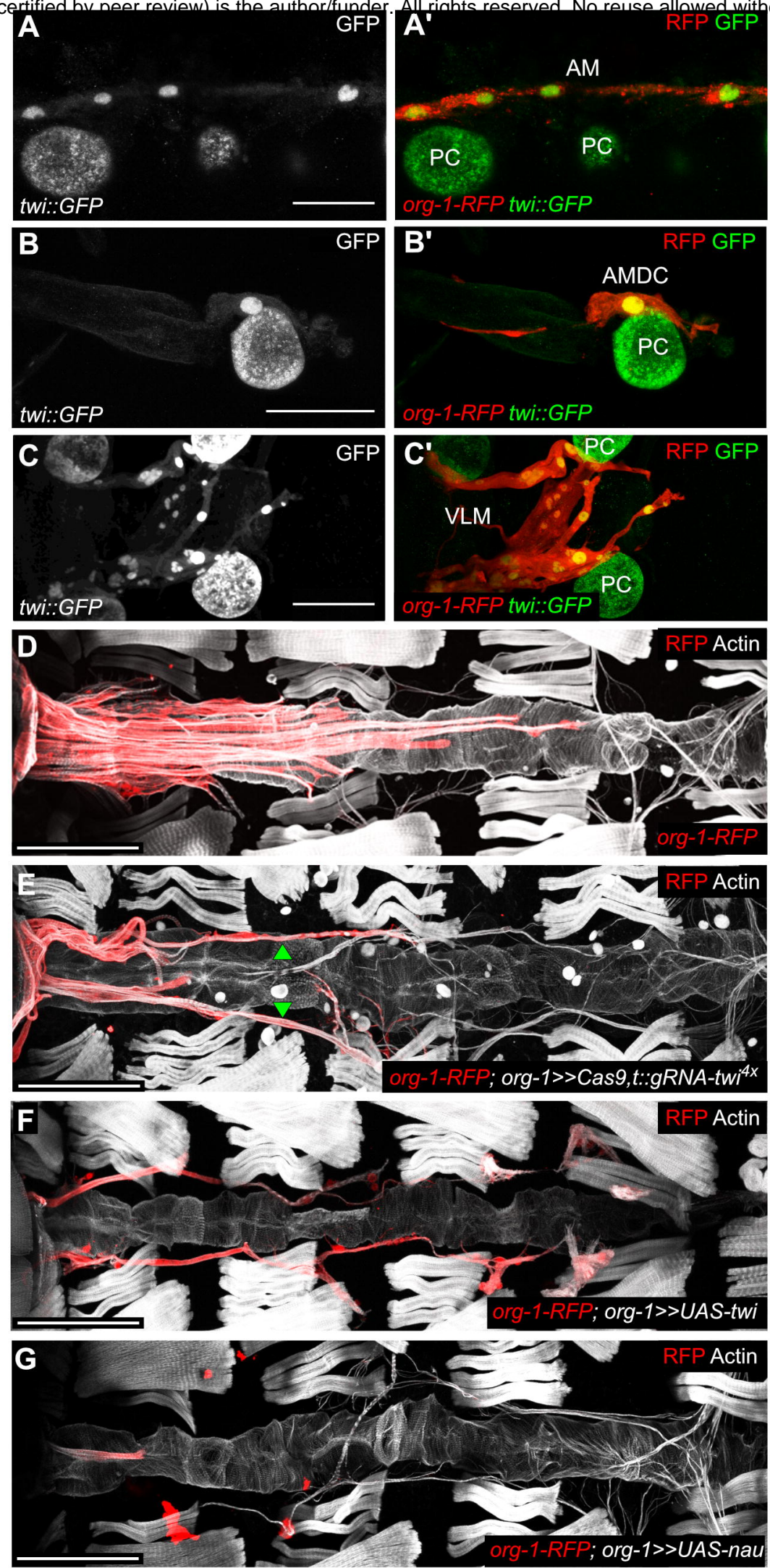

H

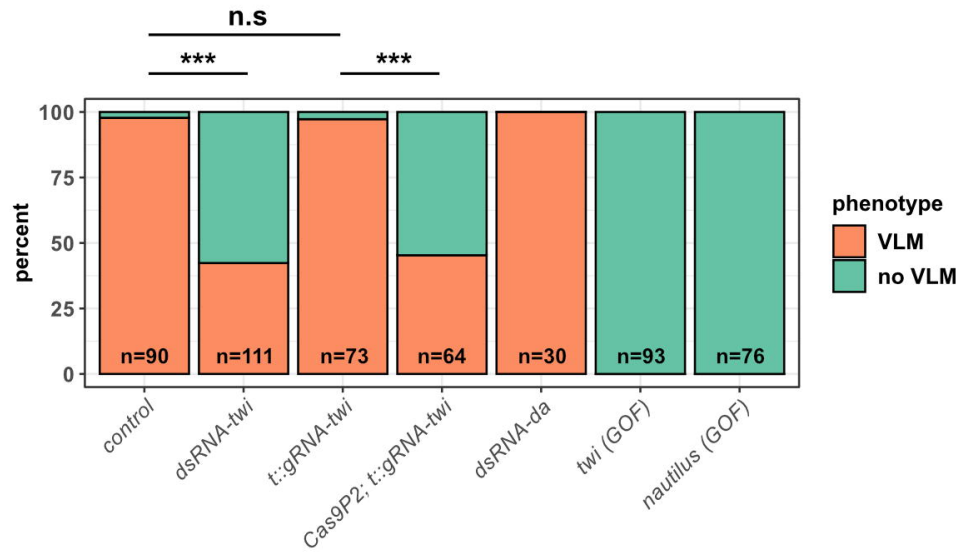



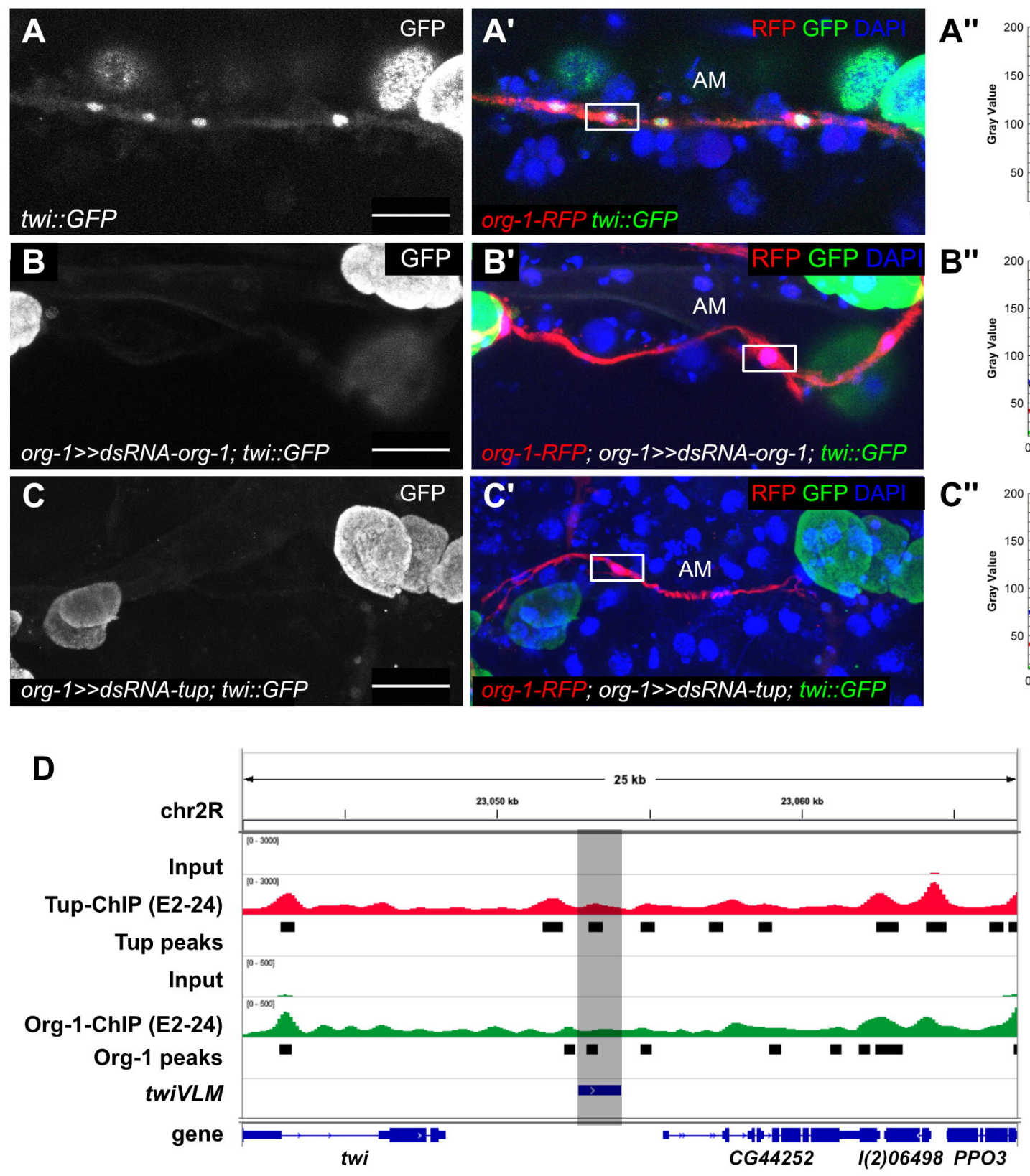
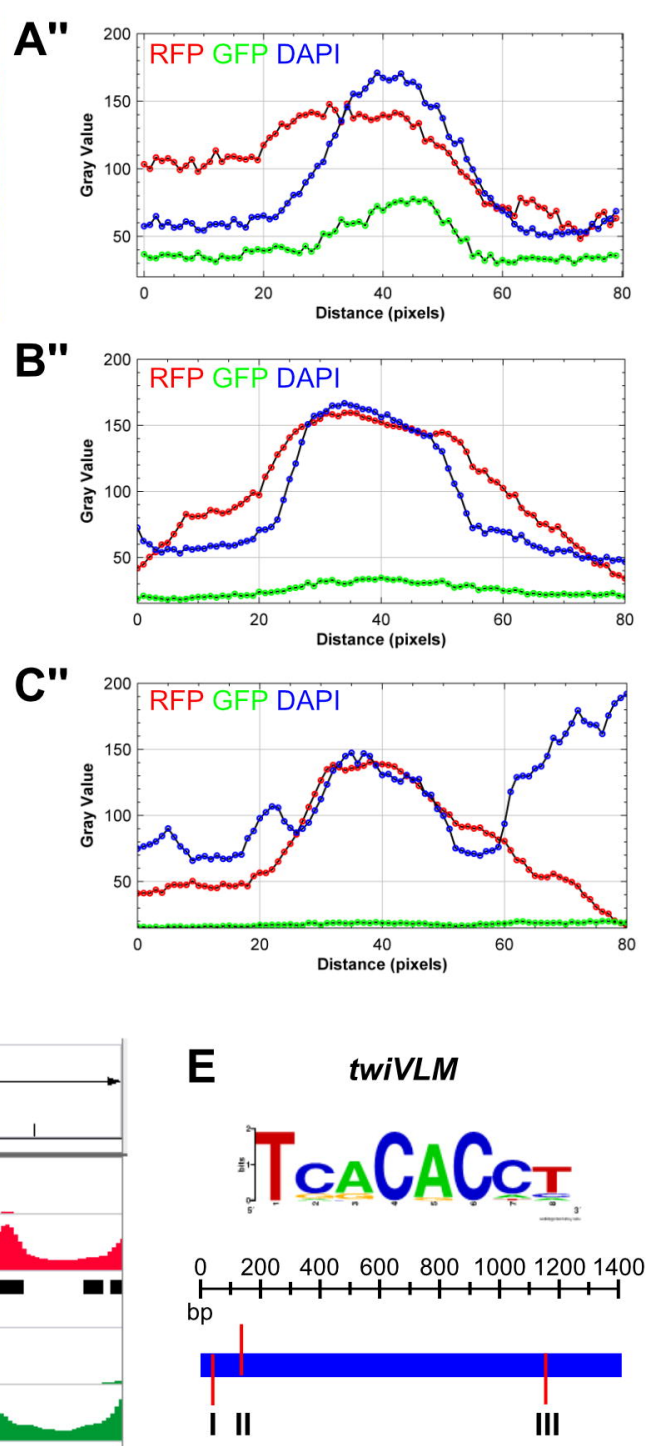

I GGAGTGAA -> GCAATTGA

II TGACACTT $\rightarrow>$ TCTAGTTT

III ACGTGTGA $\rightarrow>$ ATTAATTA

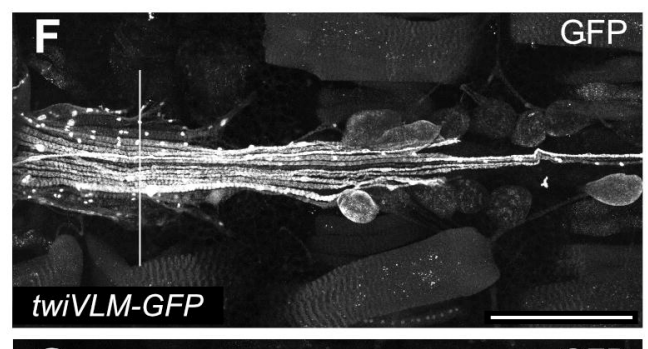

G

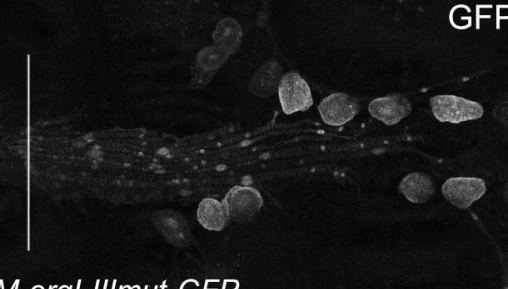

twiVLM-orgl-IIImut-GFP
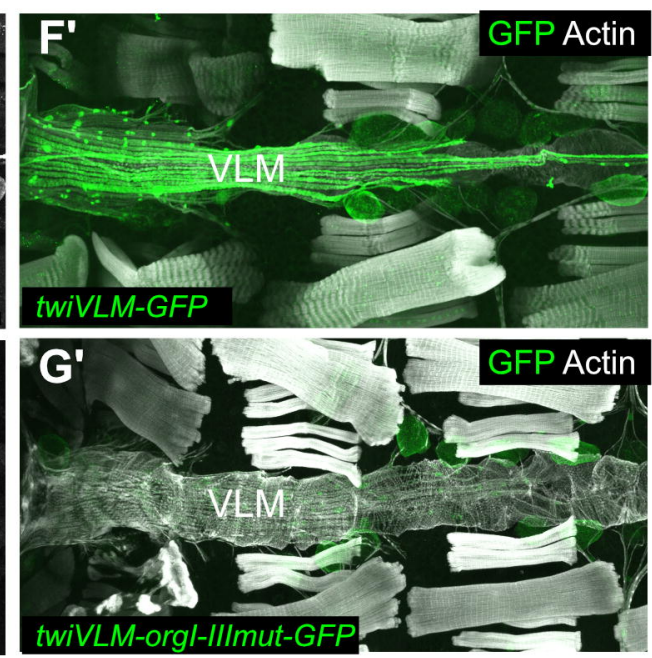

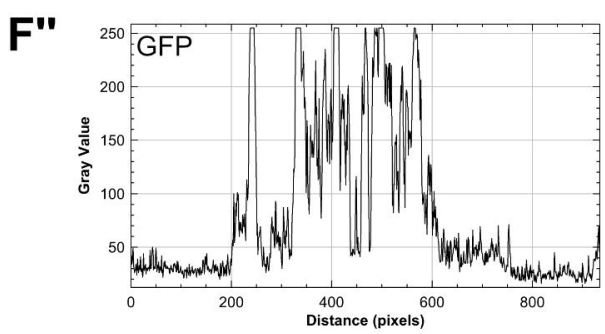

G'

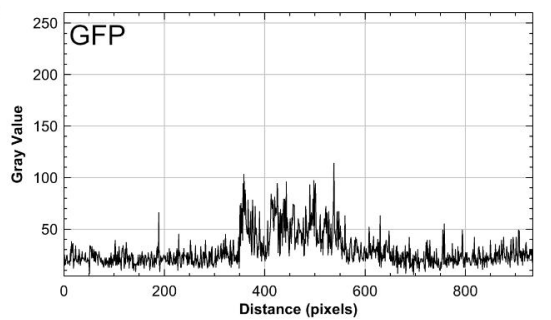


A
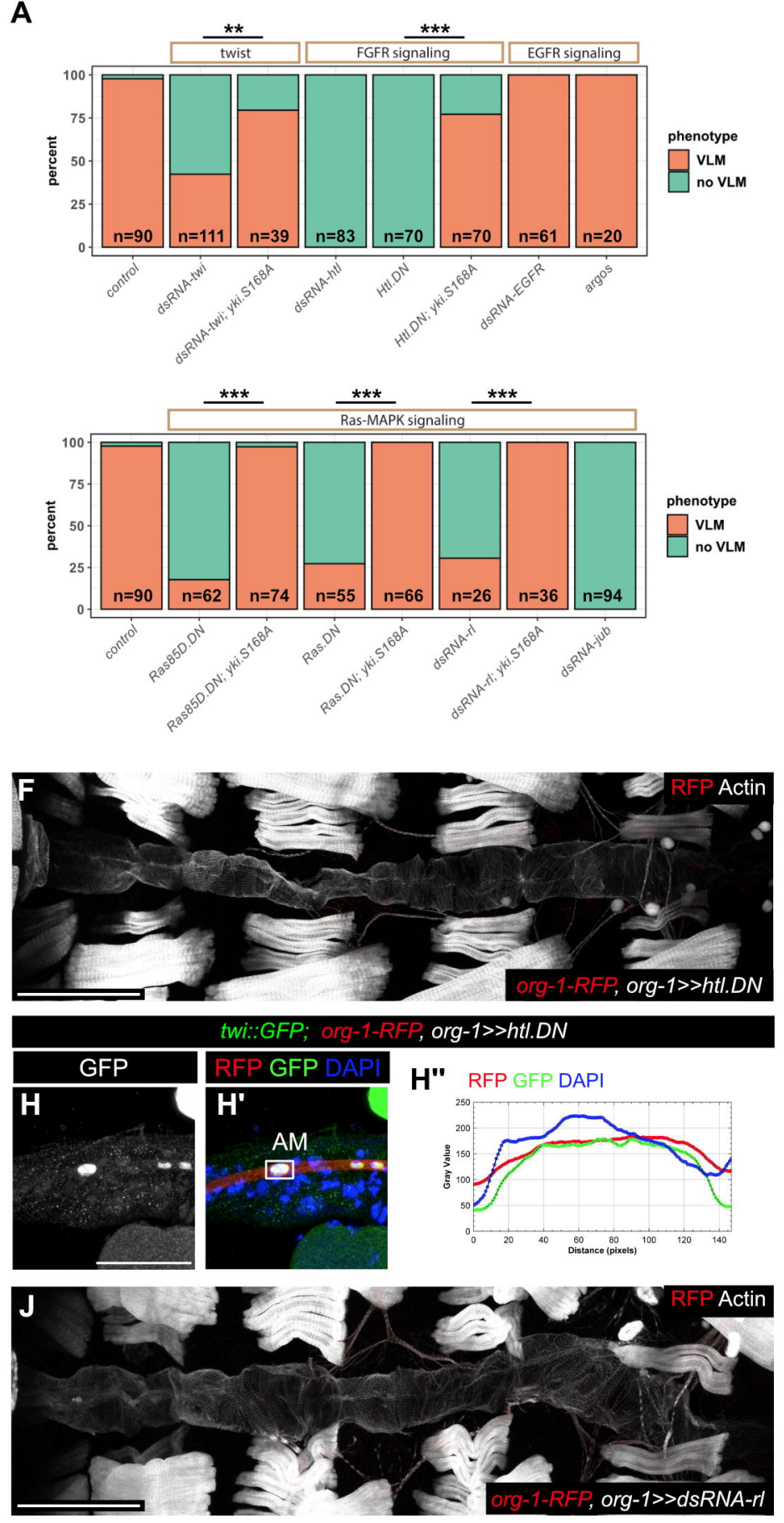

$\mathbf{L}$

alary muscle fragmentation

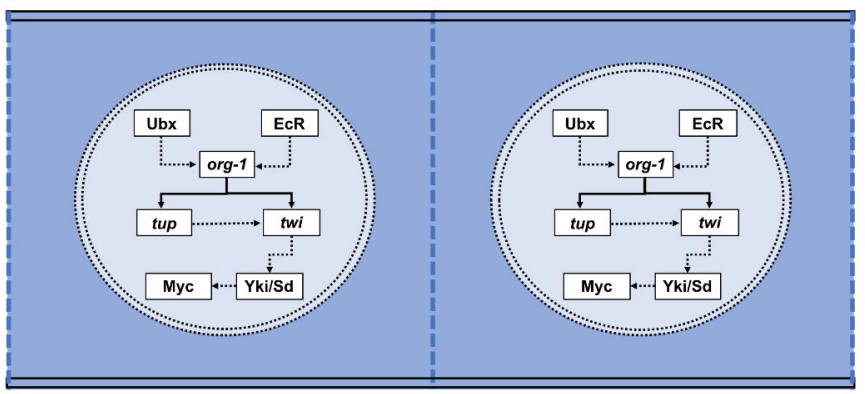

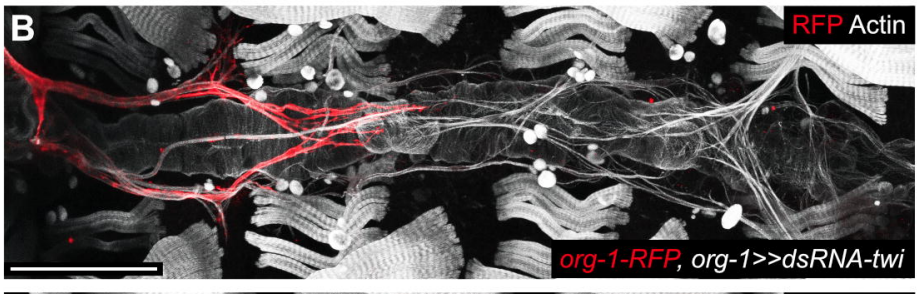
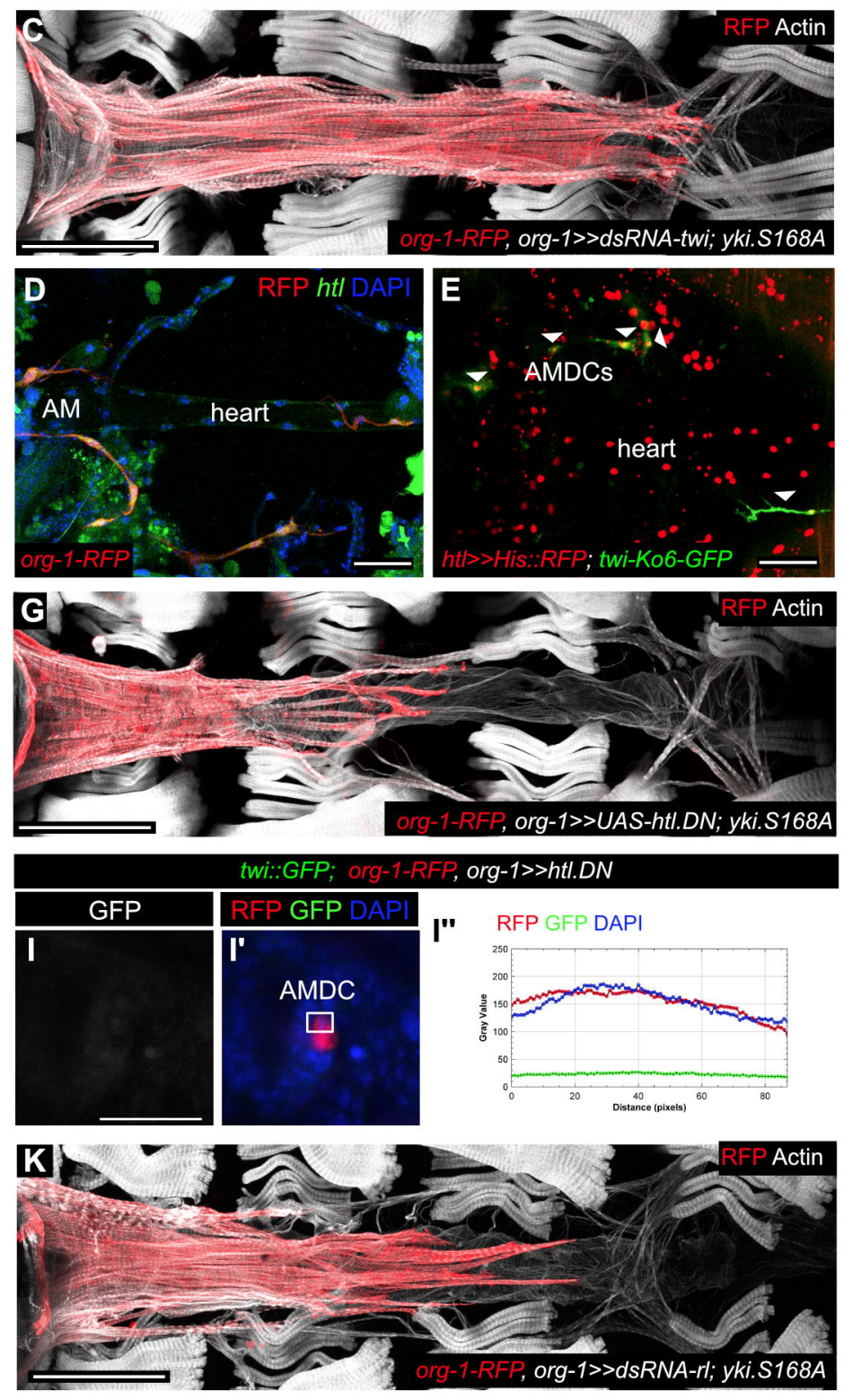

AMDC reprogramming
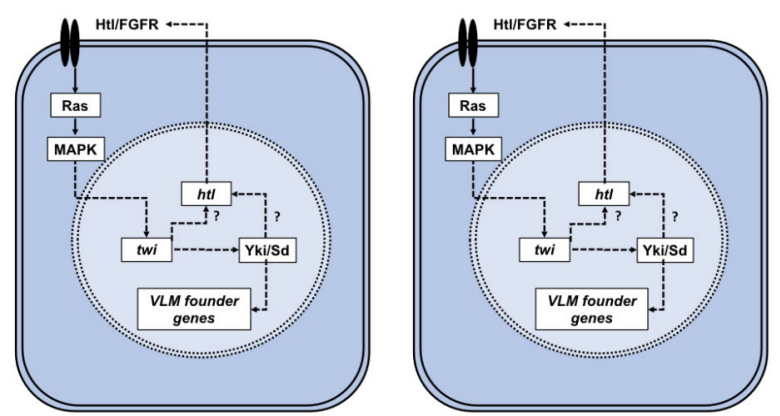